Meta

Journal des tradlucteurs

Translators' Journal

\title{
Société - association - compagnie - corporation
}

\section{Ordre des comptables agréés du Québec - Comité de terminologie française}

Volume 29, numéro 3, septembre 1984

URI : https://id.erudit.org/iderudit/002440ar

DOI : https://doi.org/10.7202/002440ar

Aller au sommaire du numéro

Éditeur(s)

Les Presses de l'Université de Montréal

ISSN

0026-0452 (imprimé)

1492-1421 (numérique)

Découvrir la revue

Citer cet article

Ordre des comptables agréés du Québec - Comité de terminologie française (1984). Société - association - compagnie - corporation. Meta, 29(3), 297-306. https://doi.org/10.7202/002440ar d'utilisation que vous pouvez consulter en ligne.

https://apropos.erudit.org/fr/usagers/politique-dutilisation/ 


\section{SOCIÉTÉ - ASSOCIATION - COMPAGNIE - CORPORATION}

\section{LE SENS ORDINAIRE DES TERMES SOCIÉTÉ ET ASSOCIATION}

Dans l'étude du terme SOCIÉTÉ, il convient de distinguer le sens donné à ce terme dans le langage courant, d'une part, et dans le langage juridique, d'autre part. Le profane donne en effet au terme SOCIÉTÉ une extension beaucoup plus grande que le juriste. Tout dictionnaire général nous dit qu'une SOCIÉTÉ est un "ensemble de personnes réunies pour une activité commune ou par des intérêts communs $\gg 1$. Cette définition laisse sous-entendre qu'une SOCIÉTÉ peut poursuivre un BUT LUCRATIF ou NON LUCRATIF. On parlera alors aussi bien de société commerciale, de société d'écrivains, de société de la Croix-Rouge, etc.

Le terme ASSOCIATION suscite les mêmes commentaires et on le définit communément comme un "groupement de personnes qui s'unissent en vue d'un but déterminé $)^{2}$. On parlera, par exemple, d'associations économiques, politiques, professionnelles, internationales, commerciales, financières, etc.

\section{LE SENS JURIDIQUE DES TERMES SOCIÉTÉ ET ASSOCIATION}

Pour le juriste, l'emploi des termes SOCIÉTÉ et ASSOCIATION est critique en raison des différences qui existent entre les divers systèmes juridiques, notamment le droit français, le droit québécois et le droit canadien (c'est-à-dire le droit fédéral et celui des provinces à l'exception du Québec).

Dans ces trois systèmes juridiques, on distingue nettement la SOCIÉTÉ de l'ASSOCIATION. Par définition, une SOCIÉTÉ découle d'un contrat par lequel deux ou plusieurs personnes conviennent de mettre en commun des biens ou leur industrie, en vue de partager les bénéfices qui pourront en résulter. En revanche, une association tire son origine d'une convention par laquelle deux ou plusieurs personnes mettent en commun leurs connaissances ou leur activité dans un but autre que de partager des bénéfices. Une société poursuit ainsi un but lucratif tandis qu'une association a essentiellement un but non lucratif. Ce qui distingue la société de l'association, ce n'est donc pas tant l'activité économique que toutes deux exercent, mais le but poursuivi, c'est-à-dire l'existence ou l'absence d'un partage de bénéfices. Souvent, dans le cas d'une association, on explicitera cette différence en parlant d'ASSOCIATION SANS BUT LUCRATIF ou de GROUPEMENT SANS BUT LUCRATIF.

\section{LA SOCIÉTÉ EST-ELLE UNE PERSONNE MORALE?}

En droit français et québécois, la SOCIÉTÉ DE PERSONNES constitue une PERSONNE MORALE qui est propriétaire des biens que les associés ou sociétaires ont investis dans la société. Outre la capacité juridique de posséder des biens, une société de personnes a également celle de conclure des contrats et d'ester en justice. Cependant, la principale caractéristique juridique d'une société de personnes consiste en ce qu'elle n'est pas entièrement indépendante des associés qui la constituent, ce qui entraîne pour ces derniers une RESPONSABILITÉ ILLIMITÉE à l'égard des dettes de la société, responsabilité qui, dans certains cas, n'est que CONJOINTE ${ }^{3}$ (sociétés civiles et, dans

1. Paul Robert (1978) : le Petit Robert, Dictionnaire alphabétique et analogique de la langue française, Société du Nouveau Littré, Paris, p. 1823.

2. Ibid., p. 116.

3. "Les associés ne sont pas tenus solidairement des dettes sociales. Ils sont tenus envers le créancier chacun pour une part égale, encore que leurs parts dans la société soient inégales. Cet article ne s'applique pas aux sociétés commerciales. " Code civil du Québec, article 1854. 
d'autres cas, est SOLIDAIRE ${ }^{4}$ (sociétés en nom collectif). La responsabilité dans une société dont l'objet est CIVIL peut être SOLIDAIRE si les associés en décident ainsi.

En droit canadien, la société de personnes n'est pas une personne morale parce que, dans ce cas, PERSONNE MORALE et RESPONSABILITÉ LIMITÉE sont deux notions qui ne peuvent être dissociées ainsi que l'indique l'expression body corporate.

Les SOCIÉTÉS DE CAPITAUX ainsi que les ASSOCIATIONS constituées en vertu de lois spéciales (au Québec, troisième partie de la Loi sur les compagnies et, au Canada, Partie II de la Loi sur les corporations canadiennes, 1964-65, c. 52, art. 2) ont une PERSONNALITÉ MORALE en ce sens qu'elles sont " titulaires d'un patrimoine collectif et d'une certaine capacité juridique sans avoir une existence corporelle ou physique ${ }^{5}$. Les ACTIONNAIRES ou ASSOCIÉS de ces sociétés de capitaux et de ces associations assument une RESPONSABILITÉ LIMITÉE qui ne va généralement pas au-delà de leur apport.

En France, les sociétés civiles et les sociétés commerciales, de personnes et de capitaux, ont, dès leur immatriculation au registre du commerce et des sociétés, la personnalité morale et, par voie de conséquence, une capacité juridique pleine et entière. En revanche, l'association a une capacité réduite, limitée par le principe dit de la spécialité, c'est-à-dire la conformité de ses actes à son objet, cette capacité étant plus ou moins grande selon que l'association est simplement déclarée ou reconnue d'utilité publique.

\section{LES SOCIÉTÉS DE PERSONNES}

La SOCIÉTÉ DE PERSONNES est un groupement de personnes (les associés) qui conviennent de mettre en commun des biens, leur crédit ou leur industrie, en vue d'atteindre un objectif donné. En droit français et québécois, les sociétés de personnes se subdivisent en SOCIÉTÉS CIVILES et en SOCIÉTÉS COMMERCIALES. Par définition, une SOCIÉTÉ CIVILE a pour objet des opérations civiles, par exemple la prestation de services comptables, financiers, jurdiques ou médicaux. En d'autres mots, une opération ayant un caractère lucratif sans être commerciale est une opération civile. Un cabinet de comptables agréés avec des associés, par exemple, constitue une société civile, à moins que les associés n'aient opté pour la responsabilité solidaire, ce qui ferait de leur société une société en nom collectif et, par le fait même, une société commerciale. En contexte québécois, on se méfiera donc de rendre systématiquement partnership par l'expression "société en nom collectif " sans s'assurer que la responsabilité est solidaire. Si l'on ne dispose pas de cette information, il est préférable de traduire partnership par SOCIÉTÉ DE PERSONNES. La SOCIÉTÉ CIVILE est une notion juridique française et québécoise qui n'a pas sa contrepartie en droit anglais.

Les SOCIÉTÉS DE PERSONNES sont dites COMMERCIALES lorsqu'elles ont principalement pour objet la fabrication d'un produit ou le commerce de marchandises. Ces sociétés sont soit des SOCIÉTÉS EN NOM COLLECTIF, soit des SOCIÉTÉS EN COMMANDITE (SIMPLE). En France, la Loi du 24 juillet 1966 sur les sociétés commerciales précise ce qui suit : "Sont commerciales à raison de leur forme et quel que soit leur objet, les sociétés en nom collectif, les sociétés en commandite simple,

4. "Les sociétés en nom collectif sont celles qui sont formées sous un nom collectif ou raison sociale, consistant ordinairement dans le nom des associés ou de l'un ou de plusieurs d'entre eux, et dans lesquelles tous les associés sont conjointement et solidairement tenus des obligations de la société. "Code civil du Québec, article 1865 .

5. Fernand Sylvain (1982) : Dictionnaire de la comptabilité et des disciplines connexes, Institut canadien des comptables agréés, Toronto, p. 57. 
les sociétés à responsabilité limitée et les sociétés par actions. "Notons ici que la notion de société commerciale existe aussi bien au Québec qu'en France.

La SOCIÉTÉ EN NOM COLLECTIF est la plus connue des sociétés de personnes. C'est, par définition, une société commerciale qui a une vocation économique. De plus, comme nous l'avons dit ci-dessus, la RESPONSABILITÉ des associés à l'égard du PASSIF SOCIAL est non seulement ILLIMITÉE mais SOLIDAIRE.

La SOCIÉTÉ EN COMMANDITE (limited partnership) est une «société de personnes constituée d'un ou plusieurs ASSOCIES appelés COMMANDITÉS ou GÉRANTS $^{6}$ (general partners) chargés de la gestion de la société et responsables indéfiniment et solidairement des dettes de la société, et d'un ou plusieurs autres ASSOCIÉS appelés COMMANDITAIRES ou ASSOCIÉS PASSIFS (limited partners) qui fournissent un apport en argent ou en nature et dont la responsabilité à l'égard des dettes de la société se limite à leurs apports dans cette dernière» ${ }^{7}$. En France et en Belgique, on trouve aussi la SOCIÉTÉ EN COMMANDITE PAR ACTIONS qui présente les mêmes caractéristiques que la SOCIÉTÉ EN COMMANDITE SIMPLE sauf que les commanditaires ont la qualité d'actionnaires.

Notons aussi l'existence aux États-Unis de la joint stock company, c'est-à-dire la SOCIÉTÉ PAR ACTIONS Ã RESPONSABILITÉ ILLIMITẼE que l'on définit comme une "ENTREPRISE SANS PERSONNALITÉ MORALE (unincorporated business) établie à la suite d'une entente entre les associés qui la constituent et qui, contrairement aux actionnaires d'une société ordinaire de capitaux, sont personnellement et solidairement responsables des dettes de l'entreprise $\$ 8$. En Angleterre et dans certaines provinces canadiennes, la joint stock company est synonyme de la limited company dont il sera question dans la section suivante.

\section{LES SOCIÉTÉS DE CAPITAUX}

La SOCIÉTÉ DE CAPITAUX (business corporation), appelée aussi SOCIÉTÉ PAR ACTIONS, est un groupement constitué en vue de réaliser un bénéfice, doté de la personnalité morale, et dont le capital est divisé en actions, chaque actionnaire n'engageant sa responsabilité que jusqu'à concurrence de son apport.

Une société de capitaux est nécessairement une SOCIÉTÉ COMMERCIALE, mais toutes les sociétés commerciales ne sont pas des sociétés de capitaux. C'est pourquoi on peut contester la justesse de la désignation actuelle (Loi sur les sociétés commerciales canadiennes) que le gouvernement fédéral a donnée en 1978 à la loi qui avait été adoptée en 1975, sous le titre de Loi sur les corporations commerciales canadiennes.

\section{SOCIÉTÉ, COMPAGNIE ET CORPORATION}

Le terme SOCIÉTÉ dans le syntagme SOCIÉTÉ DE CAPITAUX est utilisé internationalement et son emploi, dans un contexte précis, ne devrait susciter aucune difficulté d'interprétation. À plus forte raison, aucun doute ne subsistera si on lui ajoute, selon le cas, le complément voulu, c'est-à-dire de capitaux, en nom collectif, de personnes, etc. Au Québec, le législateur a, jusqu'à présent, retenu le terme COMPAGNIE (plus précisément COMPAGNIE Å FONDS SOCIAL) qui, en Europe, est archaïque lorsqu'on l'emploie pour désigner une société de capitaux, sauf dans les secteurs des assurances, du transport aérien et maritime et des services publics. Il semble que l'alignement sur le français international exigerait que le Québec refonde sa Loi sur les compa-

6. En France, il peut exister une différence entre commandités et gérants mais, à défaut de clause statutaire contraire, tous les commandités sont des gérants.

7. Fernand Sylvain, op. cit., p. 294.

8. Ibid., p. 279. 
gnies et parle dorénavant de SOCIÉTÉS. Quant au terme CORPORATION que l'on ne devrait employer que pour désigner " l'ensemble des personnes exerçant le même métier ou la même profession " 9 , le gouvernement fédéral a donné l'exemple en remplaçant, dans l'intitulé donné en premier lieu à sa loi, le terme CORPORATIONS par le terme SOCIÉTÉS. Cependant, le mot CORPORATION continue d'être incorrectement utilisé, en particulier dans la loi de l'impôt sur le revenu (Canada).

\section{LA SOCIÉTÉ À RESPONSABILITÉ LIMITÉE (S.A.R.L.) \\ ET LA SOCIÉTÉ ANONYME (S.A.)}

Ces deux expressions sont employées en France mais il serait imprudent de les utiliser au Canada à moins que la législation sur les sociétés ne soit modifiée. En France, la SOCIÉTÉ À RESPONSABILITÉ LIMITÉE (S.A.R.L.) est une société mixte se situant entre la société de personnes et la société de capitaux. En Belgique, ce type de société porte le nom de SOCIÉTÉ DE PERSONNES Â RESPONSABILITÉ LIMITÉE. Dans une S.A.R.L. aucun des associés n'est responsable au-delà de son apport mais les PARTS SOCIALES non négociables sont difficiles à céder à des tiers en raison de la procédure d'agrément et de préemption qui doit être obligatoirement suivie en cas de cession des parts. Ainsi une S.A.R.L. n'émet pas des actions mais des parts sociales qui d'ailleurs ne donnent pas lieu à l'émission de certificats.

La SOCIÉTÉ ANONYME (S.A.) est une société de capitaux dans laquelle les parts de capital sont représentées par des actions généralement transmissibles et négociables. «La société anonyme doit compter au moins sept actionnaires et disposer d'un capital social minimal si elle fait appel public à l'épargne. La loi prévoit que la société à responsabilité limitée dont le nombre d'associés dépasse cinquante doit, dans les deux ans, être convertie en société anonyme. "10 Notons que toutes les sociétés à responsabilité limitée sont des sociétés qui ne peuvent faire appel public à l'épargne. Par ailleurs, toutes les sociétés françaises dont les actions sont inscrites à la cote officielle sont des sociétés anonymes (ou des sociétés en commandite par actions), mais il existe des sociétés anonymes qui ne font pas appel public à l'épargne.

Il convient de répéter que l'on ne devrait pas parler au Canada de sociétés à responsabilité limitée et de sociétés anonymes parce que ce sont des réalités juridiques françaises. D'ailleurs, l'emploi au Québec de l'expression SOCIÉTÉ ANONYME pourrait susciter une certaine confusion en raison du sens donné à cette expression dans le Code civil du Québec qui l'utilise pour désigner une forme de sociétés de personnes qui n'a pas un nom ou une raison sociale et dont, par conséquent, l'existence n'est pas révélée aux tiers 11 .

La loi française sur les sociétés commerciales permet de stipuler une clause d'agrément dans les statuts de société anonyme, ce qui constitue l'un des aspects de la réglementation qui rapproche celle-ci de la S.A.R.L. C'est d'ailleurs pour ce type de société qu'est principalement employée l'expression de SOCIÉTÉ FERMÉE, celle de SOCIÉTÉ OUVERTE étant réservée aux groupements dont les titres font l'objet de cessions libres ; l'exemple le plus typique est celui de la société cotée ou de celle qui fait appel public à l'épargne.

LES SOCIÉTÉS OUVERTES ET LES SOCIÉTÉS FERMÉES

Sous l'influence de l'anglais, on emploie généralement au Canada les expressions «COMPAGNIE PUBLIQUE » et "COMPAGNIE PRIVÉE » pour rendre les termes

9. Paul Robert, op. cit., p. 395.

10. Fernand Sylvain, op. cit., p. 68.

11. Code civil du Québec, art. 1870. 
anglais public company et private company. Or, si les termes public sector et private sector se rendent correctement par SECTEUR PUBLIC (ensemble des Administrations relevant de l'État ou de collectivités) et par SECTEUR PRIVÉ (ensemble des entreprises appartenant à des particuliers), on ne peut parler de société " publique "et de société "privée" pour rendre les termes public company et private company. Comme une public company est " une société de capitaux dont les actions sont inscrites à la cote officielle ou se vendent sur un marché hors cote ou encore peuvent être offertes au public de quelque façon que ce soit "12, il faudrait alors parler de SOCIÉTÉ OUVERTE pour éviter de confondre cette notion avec celle de SOCIÉTÉ PUBLIQUE ou de SOCIÉTÉ À CAPITAL PUBLIC appelée au Canada SOCIÉTÉ D'ÉTAT ou SOCIÉTÉ DE LA COURONNE. En France, on parlera, dans ce dernier cas, de SOCIÉTÉ NATIONALE dont le capital provient entièrement de l'État.

Par analogie, la private company est une SOCIÉTÉ FERMÉE et sa caractéristique principale consiste en ce que ses " actions ne sont pas inscrites à la cote officielle et ne sont pas offertes en vente au public de quelque autre façon " 13 . En règle générale, une SOCIÉTÉ FERMÉE devient une SOCIÉTÉ OUVERTE lorsqu'elle décide de faire appel public à l'épargne.

La Loi sur les valeurs mobilières adoptée au Québec en 1982 utilise les expressions SOCIÉTÉ FERMÉE et SOCIÉTÉ OUVERTE. Selon cette loi, une société est FERMÉE si on y retrouve les trois conditions suivantes : 1) le nombre de ses actionnaires est inférieur à cinquante, 2) son acte constitutif restreint la libre cession de ses actions, et 3) il lui est interdit de faire appel public à l'épargne. Une société qui ne remplirait pas l'une de ces trois conditions serait une SOCIETE OUVERTE.

\section{DÉNOMINATION SOCIALE ET RAISON SOCIALE}

L'expression RAISON SOCIALE désigne le nom qu'adopte une société de personnes lorsque l'on y mentionne le nom de tous les associés, ou celui de quelques-uns d'entre eux suivi des mots ET ASSOCIÉS ou ET CIE. Lorsque le nom sous lequel une société est connue est constitué de mots, par exemple "Au royaume du bijou ", on parlera plutôt de DÉNOMINATION SOCIALE qui, dans le cas d'une société en nom collectif, peut être accompagnée de l'abréviation ENR. mise pour " enregistrée ".

La désignation sous laquelle une société par actions est connue s'appelle toujours DÉNOMINATION SOCIALE. La Loi sur les sociétés commerciales canadiennes précise que cette dénomination sociale doit comprendre soit les mots ou expressions LIMITÉE, INCORPORÉE, SOCIÉTÉ COMMERCIALE CANADIENNE ou CORPORATION, soit les abréviations LTÉE, INC., S.C.C. ou CORP. 14 De même, les règlements adoptés en vertu de la Loi sur les compagnies du Québec prévoient que la dénomination sociale doit, afin d'indiquer que « la compagnie est à responsabilité limitée, comprendre à la fin l'expression INC. ou LTÉE sauf si elle comprend déjà l'expression corporation $\gg 15$. Au point de vue linguistique, les mots LIMITÉE et les abréviations LTÉE et S.C.C. sont acceptables, mais il serait sûrement préférable que le législateur adopte les abréviations plus éclairantes et plus justes comme c'est le cas en France où l'on parle de S.A.R.L. et de S.A.

\footnotetext{
12. Fernand Sylvain, op. cit., p. 402.

13. Ibid., p. 389.

14. Loi sur les sociétés commerciales canadiennes, art. 10.1

15. Règlements concernant les raisons sociales des compagnies régies par la première partie de la Loi sur les compagnies, art. 4.
} 


\section{LES SOCIÉTÉS DE MOYENS}

La SOCIÉTÉ DE MOYENS, appelée aussi SOCIÉTÉ DE FRAIS, porte en anglais le nom de nominal partnership et se définit comme une société de personnes ayant pour objet le partage de frais communs (loyer, secrétariat, etc.) engagés par des associés travaillant individuellement ${ }^{16}$. Rien ne s'oppose à ce que les expressions SOCIÉTÉ DE MOYENS et SOCIÉTÉ DE FRAIS soient aussi utilisées dans le cas de sociétés de capitaux qui poursuivent le même objectif.

\section{DROIT CANADIEN}

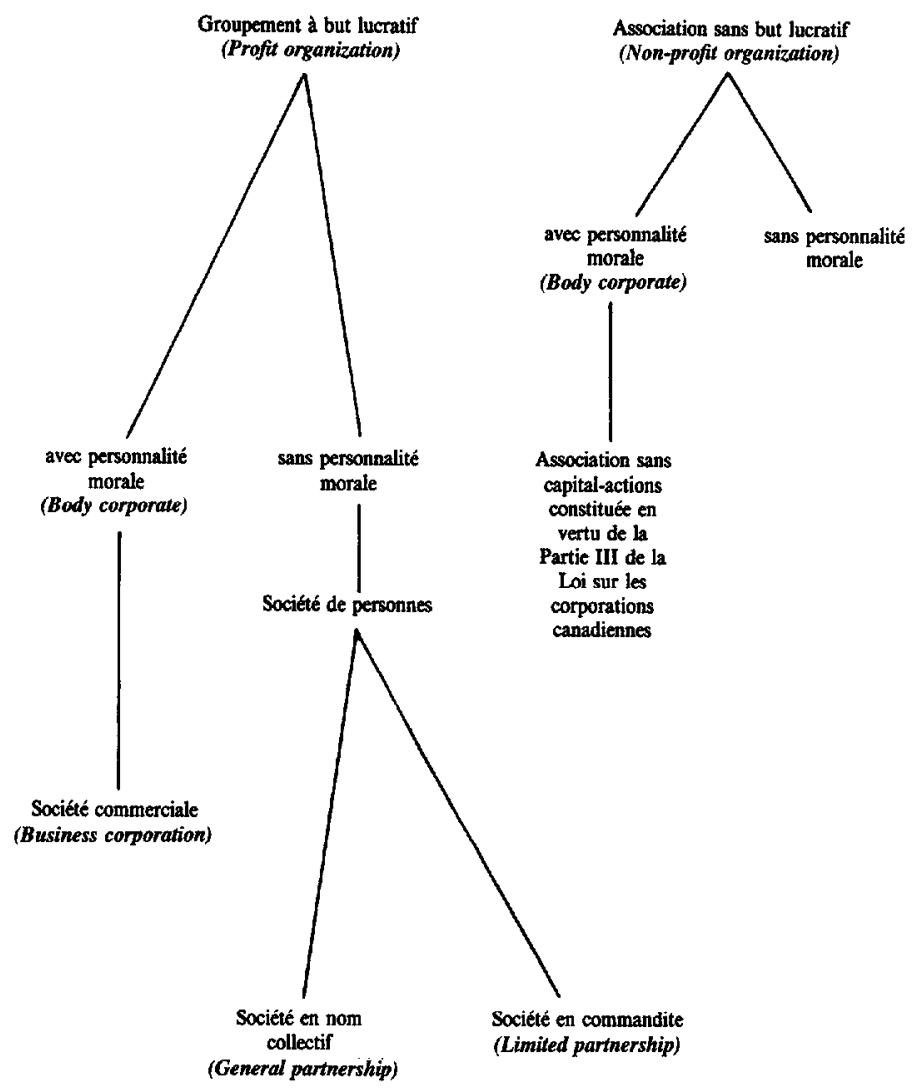

16. En droit français le terme GROUPEMENT DE MOYENS désigne, d'une manière générique, toute structure juridique dont l'objet est de mettre à la disposition de ses membres les moyens nécessaires à l'exercice d'une activité. L'article 36 de la Loi du 29 novembre 1966, relative aux sociétés civiles professionnelles, vise expressément la SOCIÉTÉ CIVILE DE MOYENS, dont l'objet est de faciliter à chacun de ses associés, nécessairement membres d'une profession libérale, l'exercice de son activité. 


\section{DROIT QUÉBÉCOIS}

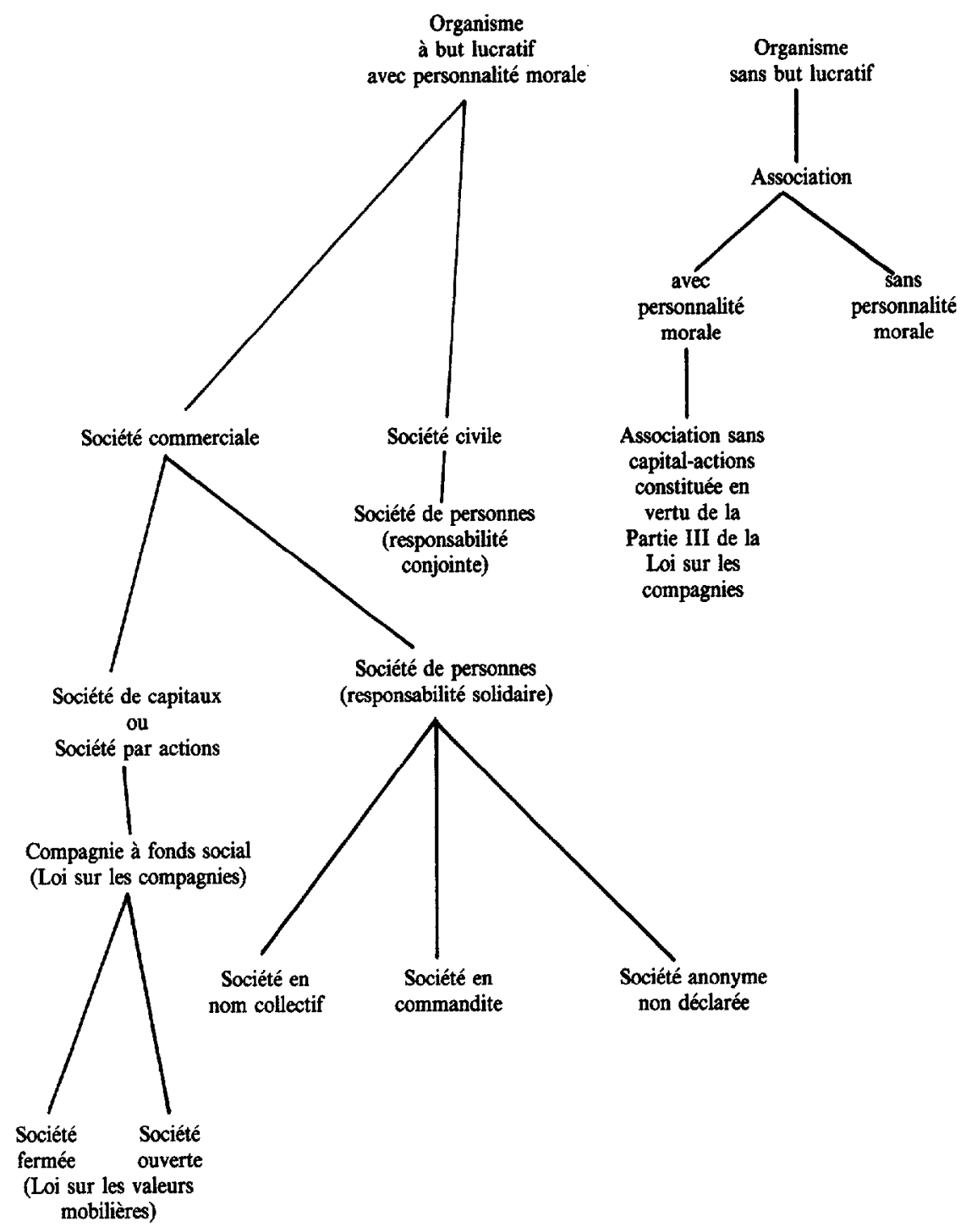




\section{DROIT FRANÇAIS}

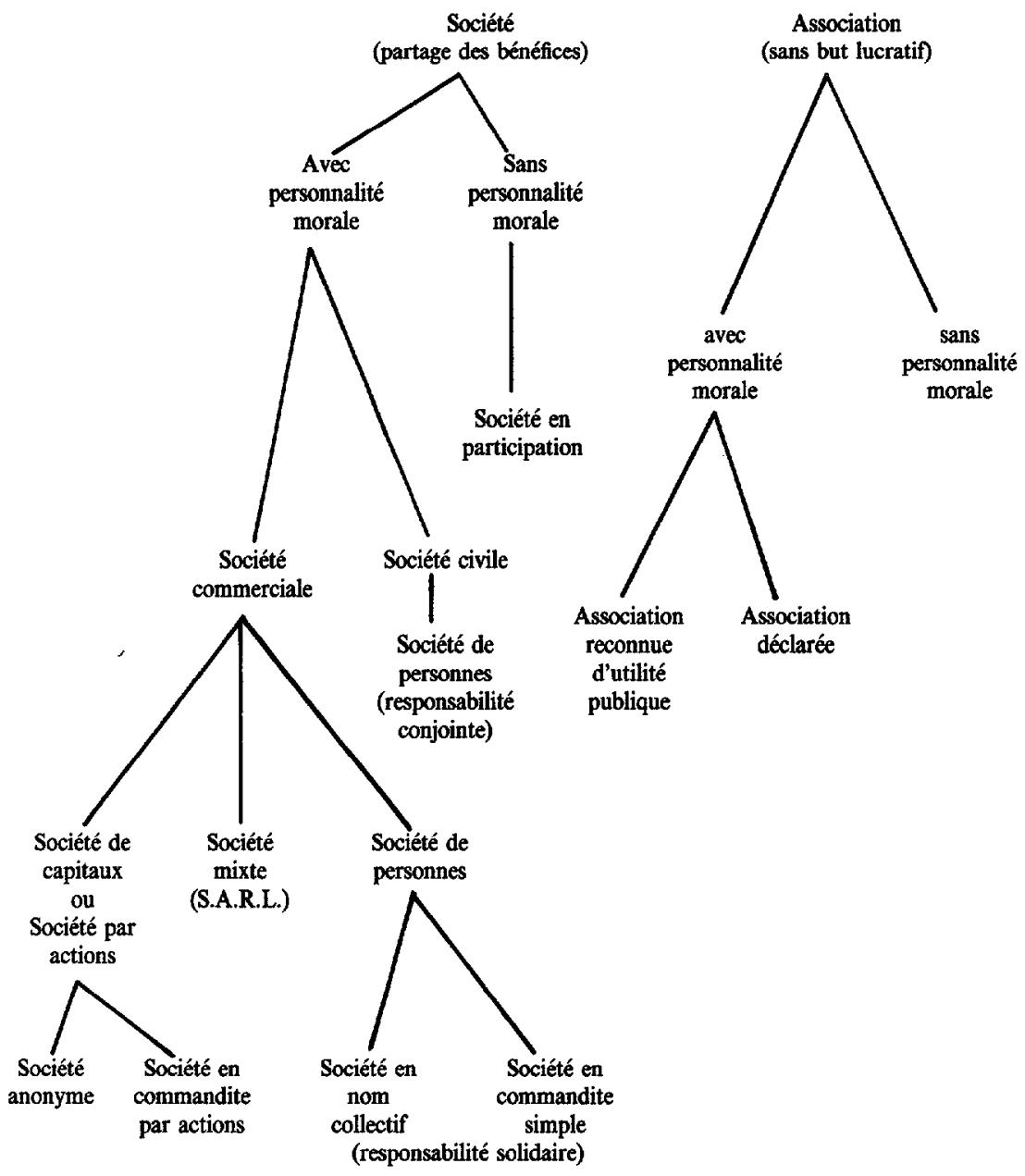




\section{DROIT BELGE}

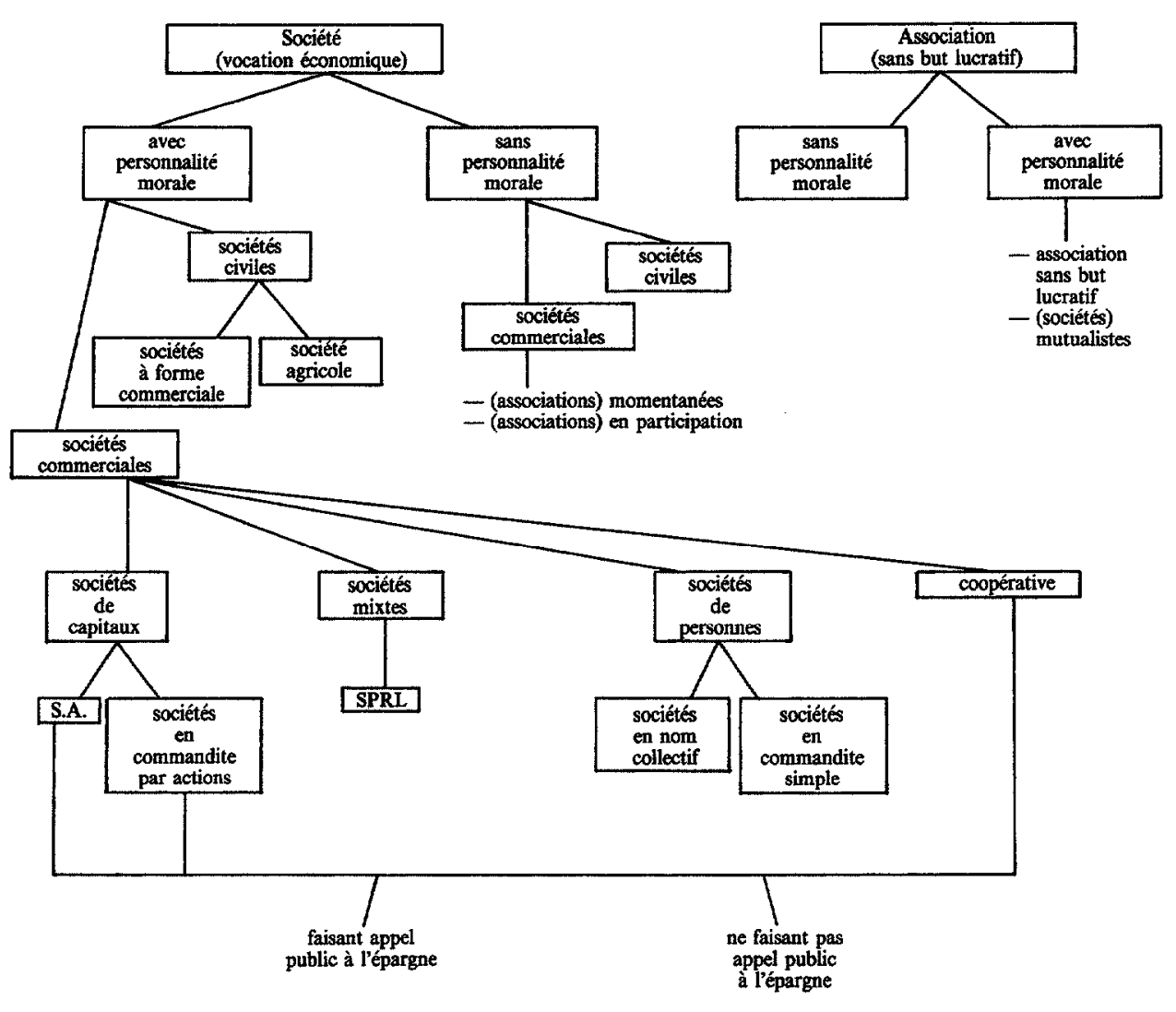




\section{LEXIQUEE}

artificial person

body corporate

business corporation

v. body corporate

personne morale

société de capitaux

société par actions

société commerciale (Can)

compagnie (à fonds social) (Québec)

société à responsabilitể limitée

(S.A.R.L.) (France)

société anonyme (S.A.) (France

et Belgique)

closely held corporation

company 1.

société

société

compagnie

association

syn. de business corporation

company 2.

corporate law

corporation 1.

corporation 2.

droit des sociétés

personne morale

syn. de business corporation

corporation (professionnelle)

ordre (professionnel)

société d'État

société de la Couronne

raison sociale (société en nom collectif)

dénomination sociale (société

de capitaux)

(associé) gérant

general partner

general partnership

incorporation

(associé) commandite

syn. de partnership

constitution en société de capitaux

société par actions à responsabilité

illimitée

syn. de business corporation

syn. de business corporation

(associé) commanditaire

assncié passif

société en commandite (simple)

société de moyens

société de frais

groupement sans but lucratif

organisme sans but lucratif

association

société de personnes

société en nom collectif (si la

responsabilité est solidaire)

société fermée

société ouverte

société publique

société à capital public

société d'Etat

société nationale

entreprise non constituée

en société de capitaux

société ouverte à grand nombre

d'actionnaires

COMITÉ DE TERMINOLOGIE FRANCCAISE ORDRE DES COMPTABLES AGRÉÉS DU QUÉBEC 